archives-ouvertes

\title{
Monitoring Local Changes in Granite Rock Under Biaxial Test: A Spatiotemporal Imaging Application With Diffuse Waves
}

Fan Xie, Yaqiong Ren, Yongsheng Zhou, Eric Larose, Laurent Baillet

\section{- To cite this version:}

Fan Xie, Yaqiong Ren, Yongsheng Zhou, Eric Larose, Laurent Baillet. Monitoring Local Changes in Granite Rock Under Biaxial Test: A Spatiotemporal Imaging Application With Diffuse Waves. Journal of Geophysical Research-Solid Earth, 2018, 123 (3), pp.2214-2227. 10.1002/2017JB014940 . hal-02376485

\section{HAL Id: hal-02376485 \\ https://hal.archives-ouvertes.fr/hal-02376485}

Submitted on 22 Nov 2019

HAL is a multi-disciplinary open access archive for the deposit and dissemination of scientific research documents, whether they are published or not. The documents may come from teaching and research institutions in France or abroad, or from public or private research centers.
L'archive ouverte pluridisciplinaire HAL, est destinée au dépôt et à la diffusion de documents scientifiques de niveau recherche, publiés ou non, émanant des établissements d'enseignement et de recherche français ou étrangers, des laboratoires publics ou privés. 


\title{
Monitoring local changes in granite rock under biaxial test: A spatio-temporal imaging application with diffuse waves
}

\author{
Fan Xie ${ }^{1,2}$, Yaqiong Ren ${ }^{1}$, Yongsheng Zhou ${ }^{2}$, Eric Larose ${ }^{3}$, Laurent Baillet ${ }^{3}$ \\ ${ }^{1}$ Key Laboratory of Seismic Observation and Geophysical Imaging, Institute of Geophysics, China Earthquake \\ Administration, Beijing 100081, China \\ ${ }^{2}$ State Key Laboratory of Earthquake Dynamics, Institute of Geology, China Earthquake Administration, Beijing 100081, \\ China \\ ${ }^{3}$ ISTerre, CNRS \& Univ. Grenoble Alpes, CS 4070038058 Grenoble Cedex 9, France
}

\section{Key Points:}

- 3-D time-lapse diffuse ultrasound tomography application of crustal rocks under complex biaxial load

- Spatiotemporal images of localized stress and deformation process

- Localized stress evolution cross-validated by full-field infrared thermography

Corresponding author: Fan Xie, xiefan@cea-igp.ac.cn 
Abstract

Diffuse acoustic or seismic waves are highly sensitive to detect changes of mechanical properties in heterogeneous geological materials. In particular, thanks to acoustoelasticity, we can quantify stress changes by tracking acoustic or seismic relative velocity changes in the material at test.

In this paper, we report on a small-scale laboratory application of an innovative time-lapse tomography technique named Locadiff to image spatio-temporal mechanical changes on a granite sample under biaxial loading, using diffuse waves at ultrasonic frequencies ( $300 \mathrm{kHz}$ to $900 \mathrm{kHz}$ ). We demonstrate the ability of the method to image reversible stress evolution and deformation process, together with the development of reversible and irreversible localized micro-damage in the specimen at an early stage. Using full-field infrared thermography, we visualize stress induced temperature changes and validate stress images obtained from diffuse ultrasound. We demonstrate that the inversion with a good resolution can be achieved with only a limited number of receivers distributed around a single source, all located at the free surface of the specimen. This small-scale experiment is a proof of concept for frictional earthquake-like failure (e.g. stick slip) research at laboratory scale as well as large scale seismic applications, potentially including active fault monitoring.

\section{Introduction}

Crustal rocks are subject to a variety of loadings such as tectonic loading, atmospheric pressure, tide and temperature [Tsai, 2011; Larose et al., 2015a]. Monitoring seismic velocity changes in rocks can provide insights into mechanical (rigidity, density etc.) evolutions associated with earthquakes [Brenguier et al., 2008; Niu et al., 2008], volcanic activity [Grêt et al., 2005; Obermann et al., 2013] or landslide destabilization [Mainsant et al., 2012]. In the industry, at ultrasonic frequencies, the same methodology addresses the demand to detect damage apparition and/or its evolution in man-made material like concrete, steel, etc. [Michaels and Michaels, 2005; Zhang et al., 2012; Planès and Larose, 2013].

The mechanical deformation of crustal rocks due to different geomechanical process do not occur homogeneously and crustal rocks have a high level of heterogeneity or granularity that may lead to localized distribution of stress and strain, potentially leading 
to crack initiation and damage development. Thus, detecting such changes and imaging their spatial distribution are of first importance. Nevertheless, such detection remains challenging since it requires ultra-high sensitive techniques, for which laboratory developments may be useful.

Many traditional laboratory approaches are invasive and destructive and can be used only once per sample, such that they are hardly suitable to explore the time-dependent velocity changes as well as micro-structure changes of the medium. Among a variety of non-destructive and/or non-invasive techniques, a bunch of recently developed full-field measurements such as X-ray tomography, Infrared Thermography (IRT) and Digital Image Correlation (DIC-2D, DIC-3D) have proved to be powerful tools to explore stress/strain fields in laboratory geodynamics [Charalampidou et al., 2014]. However, such measurements hardly reveal small changes of mechanical proprieties of the material because they are less sensitive to the state of stress, rigidity or damage due to their non-contact measurement configuration. Ultrasound techniques have long been used in the laboratory to understand the mechanics of rock deformation and are still flourishing nowadays because they are naturally and directly sensitive to the elastic properties of the material. A significant amount of work has been reported to characterize the damage evolution of rocks by means of conventional ultrasonic methods [Schubnel et al., 2006; Hall, 2009], such as ultrasonic pulse velocity or wave attenuation. Such methods are useful to assess major discontinuities associated with significant impedance contrast (or mismatch) in rocks: large cracks, cavities, and fluids. However, they have limited spatial resolution of the medium due to their low frequency.

A better spatial resolution might be achieved by increasing wave frequency. However due to heterogeneities, many polycrystalline, multi-composite crustal rocks can be considered as multiple scattering materials in the high frequency regime. This feature disables most imaging techniques. Indeed in that regime, the propagation distance between the source and the receiver is larger than the distance between two scattering events, a distance noted $\ell^{\star}$ and referred to as the scattering few mean free path. In this case direct waves are strongly attenuated and conventional methods fail to operate properly [Hirsekorn, 1982; Thompson, 1996]. On the other hand, the noise-like diffuse waves constituting the late arrivals have demonstrated not only perfect reproducibility [Snieder et al., 2002], but also high sensitivity to small changes associated with the closing and opening of pre- 
existing cracks, the development of damages at the tip of the crack, and/or with contacts at grain boundaries in heterogeneous geomaterials.

In seismology, the late arriving diffuse waves are referred to as coda waves as the tail of the seismograms $[A k i, 1969]$. Taking advantage of the sensitivity of diffuse waves that have bounced repeatedly in the medium, several methods enable monitoring tiny changes in the medium. For example, Coda Wave Interferometry (CWI) [Poupinet et al., 1984; Snieder, 2006] allows to detect relative velocity changes as low as $10^{-5}$ [Larose and Hall,
[ 2009] by measuring phase shifts. Coda Wave Decorrelation (CWD) is a similar method where one observes a loss of coherence in coda after one or several structural changes such as appearance (or disappearance, movement) of a scatterer, fluid injection or a change of geometry [Planès et al., 2014]. A downside of both CWI and CWD is that the coda wave variations provide a measurement of the material integrated over the volume of propagation, which is generally large, such that locating the changes is highly challenging.

Recently, a time-lapse differential tomography technique named Locadiff [Larose et al., 2010] was developed to explore spatio-temporal changes based on analytical diffuse sensitive kernels together with a linearized inversion technique. Although Locadiff is still an on-going project, the performance of this method was assessed through numerical studies [Pacheco and Snieder, 2005; Planès et al., 2015] and applied to imaging velocity changes and structural changes both in seismology [Obermann et al., 2013, 2014] and in Nondestructive Testing and Evaluation (NDT\&E) [Larose et al., 2015b; Zhang et al., 2016]. Using another inversion procedure, it even showed a potential to image high resolution sub-wavelength $(\sim 1 / 15 \lambda, \lambda=0.75 \mathrm{~mm})$ changes [Xie et al., 2016].

In this paper, time-lapse three-dimensional imaging of velocities and micro-cracks are operated by applying Locadiff on a natural rock sample under biaxial loading at laboratory scale. Full-field infrared thermography (IRT) is additionally used in order to crossvalidate the images of stress-induced changes measured by diffuse ultrasound. Compared with the conventional Locadiff experimental setup which uses multiple sources, a single transducer is used as a source together with eight sparsely placed receivers to cover one side of the specimen. Such setup is similar to the active source monitoring (e.g. air gun or accurately controlled routine-operated seismic source (ACROSS) experiments [Yamaoka et al., 2001; Wang et al., 2012]) at larger scale in geophysics. The aim of the paper is to employ the Locadiff technique to better understand the localized velocity changes as well 
as micro-structural changes that control the mechanical behavior of natural heterogeneous rock samples in the multiple scattering regime.

\section{Experiment setup}

\subsection{Specimen description and loading procedure}

Figure 1(a) shows the geometry of the experiment. The rock samples used in our experiments are of natural granite from Fangshan County, Southwest Beijing, China, with dimensions of $300 \mathrm{~mm} \times 300 \mathrm{~mm} \times 20 \mathrm{~mm}$. We measured the strength of the granite sample to be approximately $\sim 120-\sim 150 \mathrm{MPa}$ under uniaxial loading. A source transducer, labeled $S$, is placed in the center of the sample and surrounded by eight receivers (labeled 1 to 8). An infrared camera takes pictures of the whole top surface temperature regularly. The mechanical setup includes a biaxial loading apparatus consisting in horizontal load frames with a servo control system used to apply shear forces (Fig. 1( $a \& b)$ ). Forces are measured via two strain gauge load cells positioned inside the pressure vessel with an accuracy of $\pm 0.1 \mathrm{kN}$. Displacements are measured via linear variable differential transformers (LVDTs) with an accuracy of $\pm 1 \mu m$ referenced at the load frame and the ram. A detailed description of the experimental system is introduced in previous references [Miao et al., 2010; Collettini et al., 2014]. Once the specimen is mounted, a $750 \mathrm{kN}$ loading is carried out in both $X$ and $Y$ directions respectively to reach a $50 \mathrm{MPa}$ pressure. Around the average $50 \mathrm{MPa}$ pressure load, additionally, a $5 \mathrm{MPa}$ sinusoidal opposite-phase cyclic load pattern is applied synchronously in both directions using a digital sinusoidal waveform generator. The mechanical data (i.e. forces and LVDTs) are digitalized with a 16-bit resolution multichannel data acquisition device and stored at a sampling rate of $10 \mathrm{~Hz}$. Figure 1(c) illustrates the loading history of stress and displacement curves consisting in three successive periods of 300 seconds each (900 seconds in total).

\subsection{Data acquisition system for ultrasound}

Nine identical broad-band piezoelectric transducers (PAC ws $\alpha, 0.1-1 \mathrm{MHz}$ ) are evenly distributed every $75 \mathrm{~mm}$ over the $300 \mathrm{~mm} \times 300 \mathrm{~mm}$ area, and glued onto the bottom of the specimen. As illustrated in Fig. 1(a), the eight black transducers (labeled 1 to 8) serve as receivers while the single red transducer (labeled $S$ ) at the center serves as a source. To ensure a strong multiple scattering regime, we emit a chirp signal $s(t)$ with a frequency 
varying linearly from 300 to $900 \mathrm{kHz}$ (National Instruments PXI 5105). The amplitude of the chirp signal is $\pm 6 V$, and the duration is $0.5 \mathrm{~ms}$. The 1.5 -ms long received signals are simultaneously pre-amplified and recorded by an 8-channels 12-bit data acquisition system (National Instruments PXI 5421) at a sampling frequency of $10 \mathrm{MHz}$; the acquisitions are synchronized with the source emission signals by a $10-\mathrm{MHz}$ reference clock signals. To improve the signal-to-noise ratio (SNR), the source emission is reproduced 100 times and received records $r\left(R_{j}, t\right)\left(R_{j}\right.$ stands for receiver $j, t$ is the propagation time) were stacked accordingly.

\subsection{Data acquisition system for thermal infrared}

Figure 1(b) shows the photograph of the full-field InfraTes's ImageIR 8820 system to observe the thermal infrared changes induced by local stresses. The infrared camera with a spectrum range from 8 to $14 \mu m$ is mounted 0.5 meter above the specimen. The minimum temperature sensitivity is $25 \mathrm{mK}$, and the spatial resolution is $0.57 \mathrm{~mm}$ ( $640 \times 512$ pixels). The acquisition rate is 50 frames/second. To ensure the reliable thermal infrared observations, room temperature stabilization actions are taken during the operation (e.g. turning off lights, closing all doors, leaving curtains down and avoiding any human activity).

\section{DATA ANALYSIS}

\subsection{Diffusion characterization and sensitivity kernels}

We further correlate the received records $r\left(R_{j}, t\right)$ with the source chip signal $s(t)$ to evaluate the impulse response $h\left(R_{j}, t\right)$ in the working frequency band:

$$
h\left(R_{j}, t\right)=r\left(R_{j}, t\right) \times s(t),
$$

where $\times$ stands for the correlation.

An example of impulse response signal $h\left(R_{1}, t\right)$ acquired at receiver 1 is plotted in Fig. 2(a) together with a theoretical fit (red line). The diffusion constant can be approximated by fitting the envelop of the signal using the theoretical intensity $\sqrt{I}$ predicted by the diffusion equation. In infinite three dimensions, the diffusion equation simply reads: 


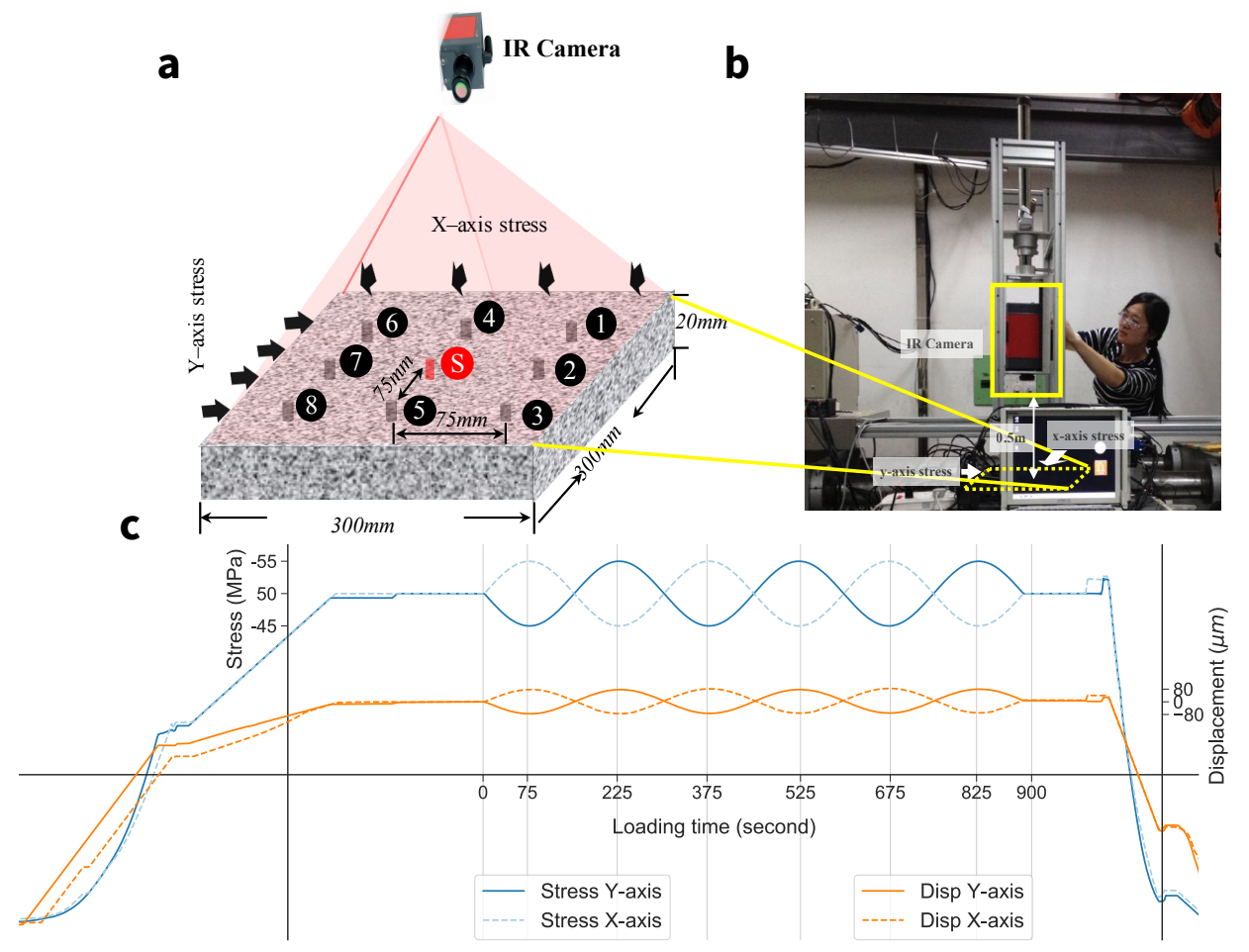

Figure 1. (a) Sketch map of the biaxial loading apparatus, sample geometry and transducers setup (black transducers labeled 1 to 8 as receivers, and one single red transducer as a source). (b) photograph of full-field infrared thermography measurement setup, the apparatus in yellow solid box is an InfraTec's ImageIR 8820 mounted 0.5-meter above the specimen; (c) loading history of stress and displacement curves consisting in three successive periods of 300 seconds each (900 seconds in total). Opposite phase cyclic loading with $5 \mathrm{MPa}$ loading amplitude oscillating around a $50 \mathrm{MPa}$ average confining pressure synchronously along the $\mathrm{X}$-axis and $\mathrm{Y}$-axis with three successive periods of 300 seconds each.

$$
I_{\infty}(S, R, t)=\frac{1}{(4 \pi \mathcal{D} t)^{3 / 2}} \exp \left(-\frac{\|S-R\|^{2}}{4 \mathcal{D} t}-\xi t\right),
$$

where $\mathcal{D}$ stands for the diffusion constant, $\xi$ is the dissipation rate (intrinsic absorption) and $\|S-R\|^{2}$ is the square of the source-receiver distance. In our case, considering the finite dimensions of the specimen, we evaluate the intensity by adding terms associated with mirror images of the source after perfect reflections on the boundaries using Sabin's principal [Sabin, 1932; Egle, 1981]. In order to evaluate the diffusivity and dissipation rate properly, we tested different source-receiver distances (from $14 \mathrm{~cm}$ to $56 \mathrm{~cm}$ ) using another sample from the same granite. By such fitting process, we evaluate roughly the average diffusion constant to be $\mathcal{D} \approx 17 \pm 8 \mathrm{~m}^{2} / \mathrm{s}$. We acknowledge a rough estima- 

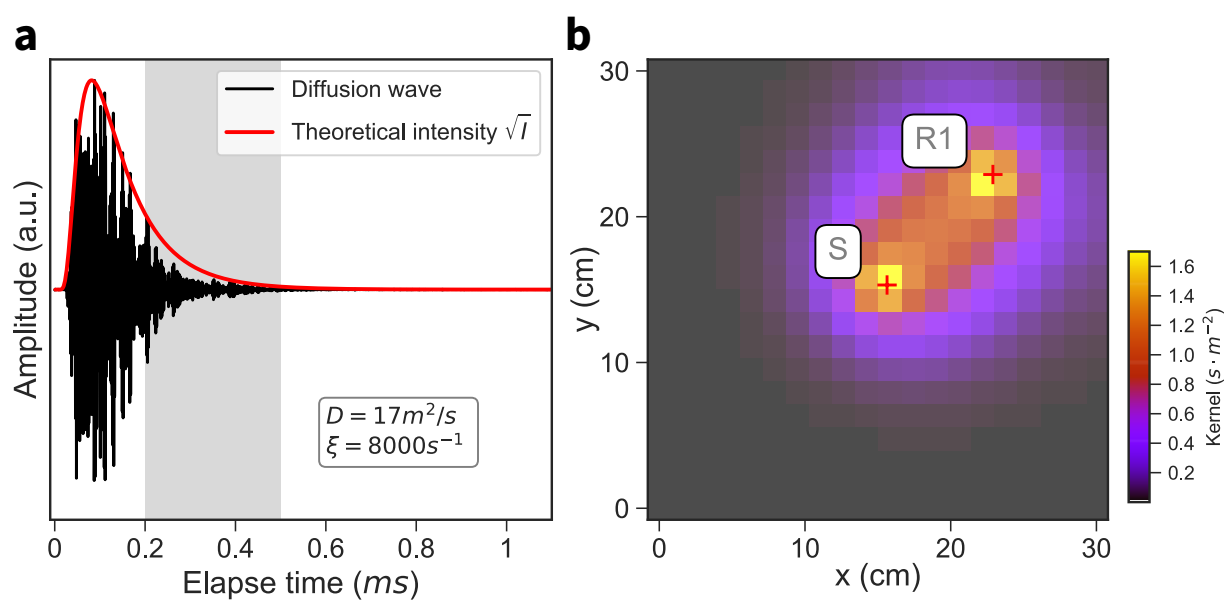

Figure 2. Illustration of a recorded diffuse ultrasound signal $h\left(R_{1}, t\right)$ and the corresponding sensitivity kernel between $S$ and $R 1$. (a) diffuse ultrasound signal and square root of it's theoretical intensity prediction using the diffusion equation with a diffusion constant $\mathcal{D}=17 \mathrm{~m}^{2} / \mathrm{s}$ and a dissipation rate: $\xi=8000 \mathrm{~s}^{-1} / \mathrm{s}$. The time-window is marked by a gray area with the center time $t=0.35 \mathrm{~ms}$ for general CWI and CWD analysis; (b) spatial distribution of the sensitivity kernel for $S$ and $R_{1}$ built with the 3-D diffusion equation considering the finite dimensions of the specimen at $0.35 \mathrm{~ms}$.

tion and assume a very large relative incertitude of $50 \%$ on this measurement. Decreasing the incertitude would request many other source-receiver distances, but as we will see later the Locadiff technique is very weakly dependent on the value of $\mathcal{D}$, so a simple order of magnitude is enough for our study.

We also assume that the energy velocity propagation is close to the shear wave velocity $c_{0} \approx 2500 \mathrm{~m} / \mathrm{s}$. The transport mean free path $\left(\ell^{\star}=3 / \mathcal{D} / c_{0}\right)$ is therefore of the order of $\ell^{\star} \approx 19 \mathrm{~mm}$, corresponding to a transport mean free time $t^{\star} \approx 8 \mu \mathrm{s}$. We summarize the physical parameters in Table 1. It is worth to note that the minimum distance $(75 \mathrm{~mm}$ ) between transducers is approximately 4 times longer than the transport mean free path $\left(\ell^{\star}\right)$, which ensures the signals to be multiply scattered and the diffusion equation to hold.

In order to predict the travel time change in the multiple scattering medium, we introduce the statistical sensitivity kernel for diffuse ultrasound (instead of predicting arrival time with a specific set of trajectories in the coda, a calculation that is hardly possible to perform). Sensitivity kernel $K\left(S, R, x_{0}, t\right)$, also called local times, represents the probability 
Table 1. Properties of diffuse ultrasound in the granite sample.

\begin{tabular}{lccc}
\hline Parameters & notation & Value & Unit \\
\hline Energy velocity & $c_{0}$ & $\sim 2500$ & $\mathrm{~m} / \mathrm{s}$ \\
Frequency range & & $300-900$ & $\mathrm{kHz}$ \\
Wavelength range & & $8-2.8$ & $\mathrm{~mm}$ \\
Diffusion constant & $\mathcal{D}$ & 17 & $\mathrm{~m}^{2} / \mathrm{s}$ \\
Transport mean free path & $\ell^{\star}$ & $\sim 19$ & $\mathrm{~mm}$ \\
Transport mean free time & $t^{\star}$ & $\sim 8$ & $\mu \mathrm{s}$ \\
\hline
\end{tabular}

of a wave emitted from location $S$ to pass at location $x_{0}$ and then to arrive at location $R$ after a period of time $t$ [Planès et al., 2014; Pacheco and Snieder, 2005]:

$$
\mathbf{K}\left(S, R, x_{0}, t\right)=\frac{\int_{0}^{t} I\left(S, x_{0}, u\right) I\left(x_{0}, R, t-u\right) d u}{I(S, R, t)} .
$$

In the above expression, $I(S, R, t)$ represents the intensity propagator for a wave to travel from $S$ to $R$ within time $t$, which simply relates to the diffusion intensity. Figure2(b) illustrates the spatial distribution of the sensitivity kernel $K$ between the source (S) and the receiver 1 at $t=0.35 \mathrm{~ms}$.

\subsection{Coda Wave Interferometry and Coda Wave Decorrelation}

We perform coda wave interferometry (CWI) and coda wave decorrelation (CWD) analysis to extract velocity changes and structural changes, respectively. We adopt the stretching method [Hadziioannou et al., 2009] because of its robustness against the noises. The signal records are noted as $h_{i}\left(R_{j}, t\right)$, where $i$ is the number of the record along the loading history. Note that there are different time scales: time $t$ is the time of the ultrasonic record (of the order of microseconds to milliseconds) and date $i$ refers to the loading history (several seconds to hundreds of seconds).

The stretching procedure is in two steps: assuming $h_{1}\left(R_{j}, t\right)$ as the reference record, (1) the current record $h_{i}\left(R_{j}, t\right)$ is stretched using interpolations with various stretching factors $\epsilon_{k}$; (2) each stretched signal $h_{i}\left(R_{j}, t\left(1+\epsilon_{k}\right)\right)$ is then compared to the reference record $h_{1}\left(R_{j}, t\right)$ by computing its correlation coefficient $C C\left(\epsilon_{k}\right)$ within a given time-windows $\left[\begin{array}{ll}t_{1} & t_{2}\end{array}\right]$ : 


$$
C C\left(\epsilon_{k}\right)=\frac{\int_{t 2}^{t 1} h_{1}\left(R_{j}, t\right) h_{i}\left(R_{j}, t\left(1+\epsilon_{k}\right)\right)}{\sqrt{\int_{t 2}^{t 1} h_{1}\left(R_{j}, t\right)^{2} d t \int_{t 2}^{t 1} h_{i}\left(R_{j}, t\left(1+\epsilon_{k}\right)\right)^{2} d t}}
$$

In practice, we choose the time-window $\left[t_{1} t_{2}\right]$ duration greater than $\geqslant 10 t^{\star}$ and SNR $\geqslant 40 d B$ which insures the stable measurements of the cross-correlation coefficients.

The stretching computation can be repeated for a huge number of values of the parameters $\epsilon_{k}$ over the plausible range of interest. This simple linear grid search algorithm is nevertheless quite inefficient, as it typically requires more than $10^{5}$ iterations. We improve the computation by using a collapsing grid search algorithm which significantly reduces the iterations to less than $10^{2}$, as an initial coarse grid is first used for the minimum misfit correlation coefficients.

The parameter $\epsilon_{\max }$ which maximizes the $C C\left(\epsilon_{k}\right)$ corresponds to the actual relative velocity change $d v / v=-\epsilon_{\max }$, micro-structural change $(d c)$ is measured from the residual waveform correlation $C C: d c=1-C C\left(\epsilon_{\max }\right)$.

\subsection{Inversion algorithm}

Before the inversion, we discretize the specimen into 800 elementary cubic cells $\Delta V$, the size of each cell $\Delta V=0.015 m \times 0.015 m \times 0.01 \mathrm{~m}$. We choose the mesh size slightly smaller than the transport mean free path. We introduce a general linear model in matrix form used to image the local changes:

$$
\mathbf{d}=\mathbf{G m}
$$

where $\mathbf{d}$ is a vector corresponding to the velocity change or the decorrelation measured for a given source-receiver pair at time $t ; \mathbf{G}$ is a matrix corresponding to the sensitivity kernel $\mathbf{K}$ at time $t$ and weighted by the cell volume, then either divided by the time for CWI $\left(\mathrm{G}=\frac{\Delta V}{t} \mathbf{k}\right)$ or multiplied by the energy velocity $c_{0}$ for CWD $\left(\mathrm{G}=\frac{c_{0} \Delta V}{2} \mathbf{k}\right) ; \mathbf{m}$ is the vector we wish to inverse that corresponds either to the relative velocity change per volume, or to the micro-structural changes characterized by the density of effective scattering cross-section changes $\sigma\left(\mathrm{m}^{2} / \mathrm{m}^{3}\right)$.

Equation 5 can not be solved directly because it is not an even-determined problem. To reduce the negative influence lead by an ill-posed problem, 32 values of CWI (resp. 

CWD) measurements from multiple time-windows ranging from $0.2 \mathrm{~ms}$ to $0.7 \mathrm{~ms}$ with $\mathrm{SNR} \geq 40 \mathrm{~dB}$ are calculated together with a linear least-square inversion solution proposed by Tarantola and Valette [1982]; Tarantola [2006] to derive the model of m:

$$
\mathbf{m}=\mathbf{m}_{0}+C_{m} G^{T}\left(G C_{m} G^{T}+C_{d}\right)^{-1}\left(\mathbf{d}-G \mathbf{m}_{0}\right)
$$

where superscript ${ }^{T}$ is the matrix transpose, $\mathbf{m}_{0}$ is the initial model filled with zeros since there is no a priori information about the perturbation and its impact such as stress distribution or about the value obtained from previous loading time, $C_{m}$ and $C_{d}$ are both diagonal covariance matrix. $C_{d}$ describes the standard deviations on measured changes in coda. For CWD, we use empirical model proposed by Planès et al. [2015] $C_{d i i}=0.3 d c$ (ii stands for each element alone the diagonal of $C_{d}$ ). For CWI, we use theoretical model proposed by Weaver et al. [2011] $C_{d i i}=\frac{1-c c_{i}^{2}}{2 c c_{i}} \frac{6 \sqrt{\pi / 2}}{f \Delta\left(2 \pi f_{c}\right)\left(t_{2}^{3}-t_{1}^{3}\right)}$, where $f_{c}$ and $f_{\Delta}$ are the center frequency and the frequency bandwidth of emitted source signal.

$C_{m}$ describes the deviations of real model from the a priori information which can reduce the under-determination of the problem. We use exponential correlation between the cells proposed by Hansen [Hansen, 1992]:

$$
C_{m i i}=\left(\operatorname{std}_{m} \frac{L_{0}}{L_{c}}\right) \exp \left(-\frac{\left|x_{i}-x_{j}\right|}{L_{c}}\right)
$$

where $s t d_{m}$ is the a priori standard deviation of the observed data $\mathbf{m} .\left|x_{i}-x_{j}\right|$ is the distance between two cells. $L_{0}=0.02 \mathrm{~m}$ is the regularization distance for which diffusive sensitivity kernels could be separated. $s t d_{m}$ and $L_{c}$ can be chosen using the $L$-curve method based on an optimal trade-off between the regularization of $\mathbf{m}$ and the quality of the fit that it provides with the d. Note that in order to inverse micro-structural change, nine iterations are carried out using Eq. 6 to constrain positive values since negative values have no physical means.

To confirm the validity of the inversion model, we perform quality tests for the model resolution $\mathbf{R}$ :

$$
\mathbf{R}=C_{m} G^{T}\left(G C_{m} G^{T}+C_{d}\right)^{-1} G
$$


The closer to one the restitution index which sum over the elements of the rows the resolution matrix $\mathbf{R}$, the more accurate the changes can be recovered by the inversion.

\subsection{Data processing for thermal infrared camera data}

We assume that the temperature recorded by the thermal infrared system originates from three contributions: (1) the physical phenomenon under study, which in our case is associated with applied stresses; (2) the thermal bias due to environmental room temperature fluctuations, such as human activities, lights, electronics etc; and (3) the experimental bias, which refers to additional fluctuations of the apparatus, such as temperature drift of the camera and noise from the data acquisition system. To obtain temperature changes induced by applied stresses as well as to improve the spatial resolution, two procedures have been applied: (1) environmental temperature changes are removed by subtracting the temperature of a reference specimen; (2) experimental bias can be reduced by spatially subtracting a reference image and applying a neighborhood average smoothing method (20 pixels $\times 20$ pixels) in the space domain and the adjacent average smoothing method in the time domain. The temperature sensitivity in this way can be improved from $25 \mathrm{mK}$ down to $\sim 5 \mathrm{mK}$, which is enough to detect $5 \mathrm{MPa}$ loads assuming a stress sensitivity coefficient of $1.03 \mathrm{mK} / \mathrm{MPa}$ [Ren et al., 2017].

\section{Experimental results}

\subsection{Velocity and micro-structural changes from each receivers}

The general evolution of the velocity changes $(d v / v$ from CWI) and the microstructural changes ( $d c$ from CWD) from each receiver as a function of loading time is illustrated in Fig. 3. We divide the receivers into two columns because the $d v / v$ and $d c$ diverge from one receiver to another at different loading times. The left column in Fig. 3 consists in receivers R2, R7, R4 and R5 located at four sides of the specimen ; the right column in Fig. 3 consists of 4 receivers of R1, R3, R6 and R8 located along the diagonals of the specimen.

Generally, the velocity changes show variations that are consistent with the local state of stress, in agreement with acousto-elasticity [Murnaghan, 1951]: the velocity increases by about $0.02 \%$ where the stress is increased by $5 \mathrm{MPa}$, and decreases by about $-0.02 \%$ where the stress is released by $5 \mathrm{MPa}$. For instance, at receiver R2 and R7 (resp 


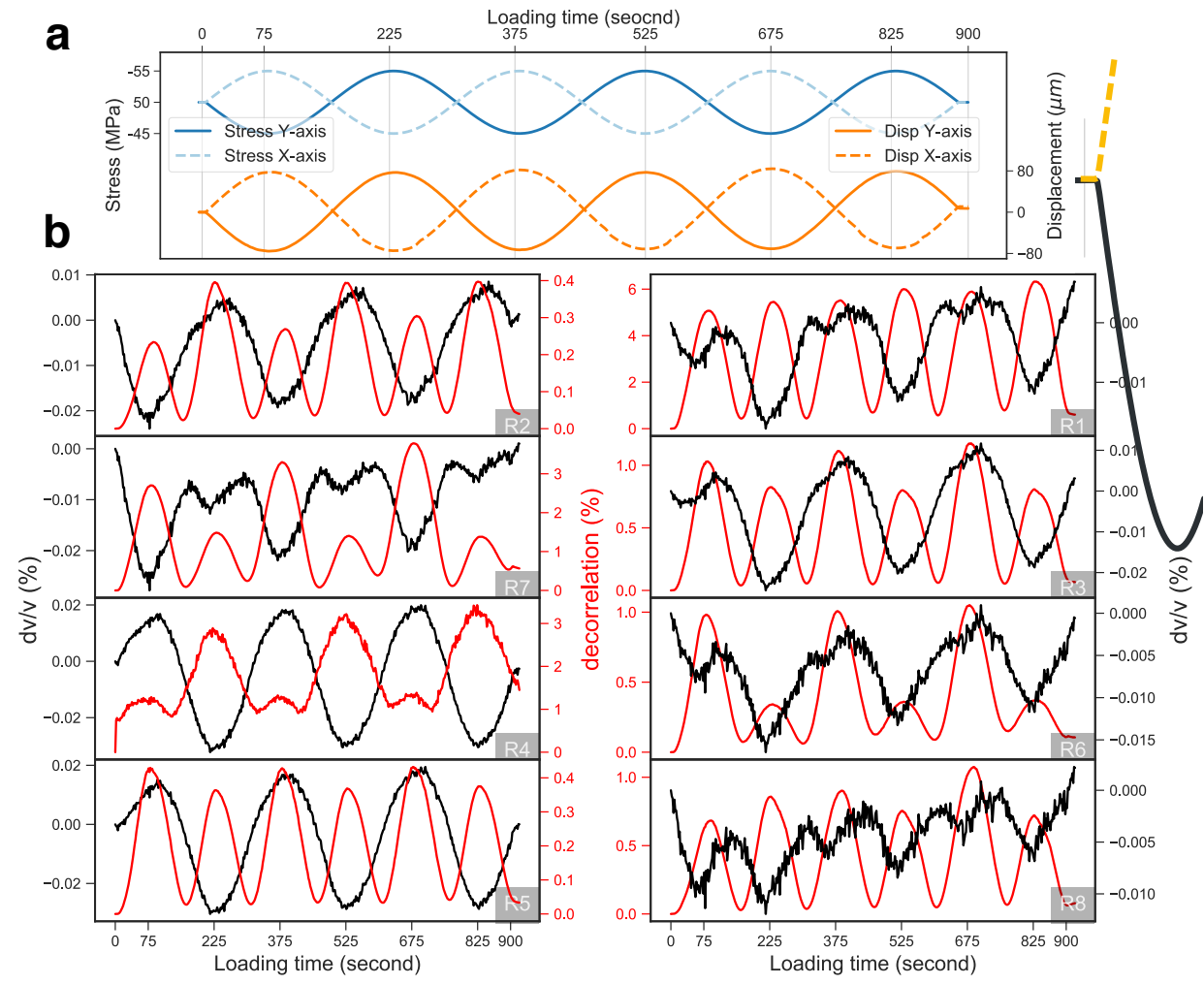

Figure 3. General evolution of $d v / v$ and $d c$ as a function of loading time. (a) The loading history of stress and displacement curves; (b) left column: velocity changes ( $d v / v$, black line) and micro-structural changes ( $d c$, red line) as a function of loading time located at four sides of the specimen (R2, R7, R4, R5); right column: velocity changes ( $d v / v$, black line) and micro-structural changes ( $d c$, red line) as a function of loading time located at four corners along the diagonals of the specimen (R1, R3, R6, R8).

$\mathrm{R} 4$ and R5), the acoustic velocity is maximum when the Y-axis (resp. the $\mathrm{X}$-axis) stress is maximum at time $225 \mathrm{~s}, 525 \mathrm{~s}$ and $825 \mathrm{~s}$, (resp. $75 \mathrm{~s}, 375 \mathrm{~s}, 675 \mathrm{~s}$ ) and the velocity is minimum when the Y-axis (resp. $\mathrm{X}$-axis) stress is minimum.

The decorrelation $(d c)$ of 8 receivers increase twice at each period of sinusoidal loading time. This is simply related to the reference state to which current waveforms are compared: the reference waveform is the first one obtained an intermediate loading, both $50 \mathrm{MPa}$ in $X$ and $Y$ direction. Any decrease or increase in stress induces an increase of decorrelation, thus making two $d c$ oscillations every one loading period. The maximum decorrelation value appears along the diagonals $(>1 \%)$ which suggest that the microdeformation (and potentially micro-damage or micro-crack initiation) is more important along the diagonals of the specimen. 
We note that "noises" are observed in the black curves that stand for the relative velocity changes at each sensor. This is attributed to the machine effect because the accuracy of the force generator $( \pm 0.1 \mathrm{kN})$ controlled by the servo control system is not high enough to stabilize such $5 \mathrm{MPa}$ opposite phase cyclic loading. It produces tiny force perturbations which are recorded by highly sensitive diffuse ultrasound during the cyclic load process. We also note that room temperature stabilization actions have been taken to minimize environmental temperature change $\left(\sim 0.2{ }^{\circ} \mathrm{C}\right)$ during the experiment, therefore we attribute the apparent relative velocity changes to the stresses applied by biaxial loading.

Based on these preliminary observations, we conclude that the CWI successfully detects the stress evolution. It suggests a non-uniformed stress distribution, which seems natural in such asymmetric loading pattern since the velocity changes along the X-direction (top and bottom areas) of the specimen are totally opposite against the Y-direction (left and right areas). CWD is also observed to have quite heterogeneous distribution. CWD detects an increase in micro-cracks and/or micro-deformation for both positive and negative loadings (with respect to the average value of $50 \mathrm{MPa}$ ) with maximum values appearing along the diagonals of the specimen. However, both CWI and CWD provide only the measurement of diffuse waves integrated over the volume where the wave actually propagated. For a proper cartography of relative velocity changes and/or local wave decorrelation, results from the inversion procedure described earlier are presented in the next section.

\subsection{Inversion resolution}

Given that the thickness of the sample is relatively small compared to other dimensions, we consider only the average value of three dimensional $d v / v$ (or $d c$ ) along $\mathrm{Z}$ to produce top-view two dimensional images in the X-Y plane. Figure 4 illustrates the inverted model distributions of changes in the X-Y plane occurring at $75 \mathrm{~s}$ loading time for the given inversion parameters. Figure 4(a) is the two dimensional image of microstructural changes characterized by the density of effective scattering cross-section changes $\sigma$. Figure 4(b) and (c) illustrate the search for the optimal inversion parameter $s t d_{m}=$ 0.43 using the L-curve test for a given value of spatial smoothing of $L=3 L_{0}$ and the restitution index of $\mathbf{R}$ which qualifies the inversion quality of micro-structural changes. Figure $4(d)$ is the two dimensional image of velocity change at 75 s loading time. Figures 4(e) and (f) illustrate the search for the optimal inversion parameter $s t d_{m}=0.048$ 

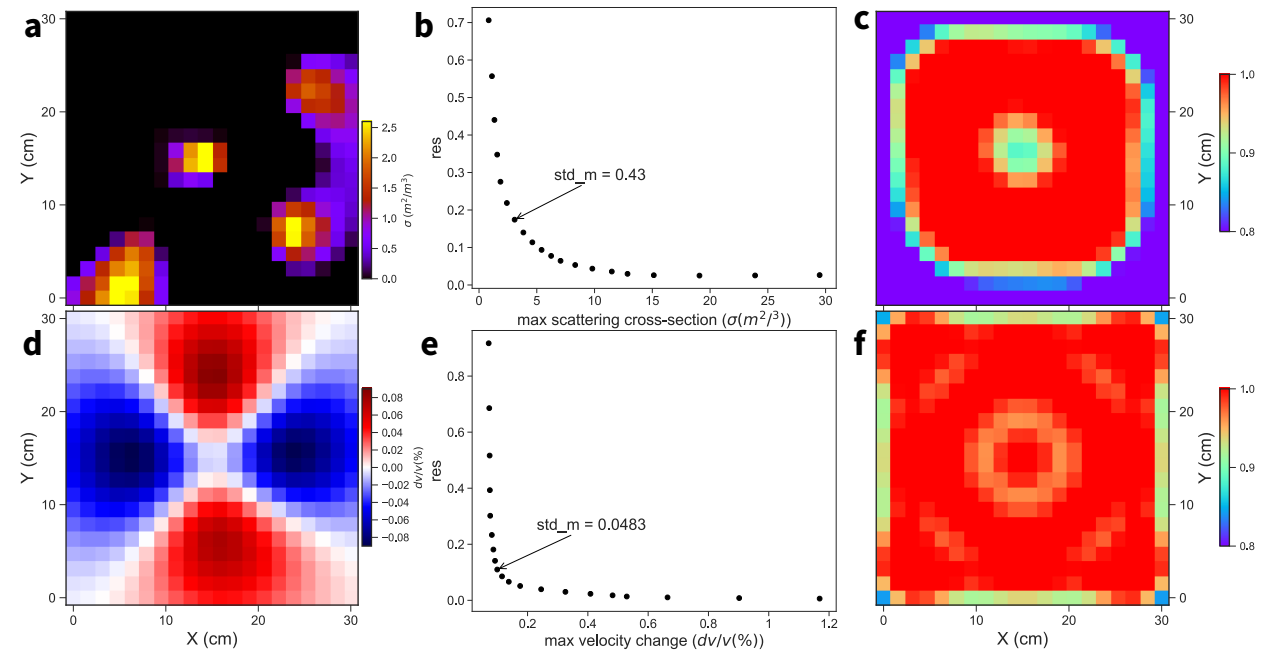

Figure 4. Images of changes occurring at $75 \mathrm{~s}$ of loading time in the $\mathrm{X}-\mathrm{Y}$ plane. (a) the distribution of micro-structural changes $(\sigma)$. (b) L-curve test for a given value of spatial smoothing of $L=3 L_{0}$. The best compromise is obtained at the corner of the slop, at the arrow for $s t d_{m}=0.43$. (c) Resolution index map of $\sigma$ which value is relatively homogeneous in this plane, with values greater than 0.8 . (d) Distribution of velocity change $(d v / v)$. (e) L-curve test for a given value of spatial smoothing of $L=3 L_{0}$. The best compromise is obtained at the corner of the slop, at the arrow point for $s t d_{m}=0.048$. (f) Resolution index map of $d v / v$, which is relatively homogeneous in this plane, with values greater than 0.8 .

using the L-curve test for a given value of spatial smoothing of $L=5 L_{0}$ and the restitution index of $\mathbf{R}$ which qualifies the inversion quality of velocity changes. Both the restitution indexes of micro-structural and velocity are greater than 0.8 in the central area of the images, indicating that we are able to inverse the changes with confidence. It also indicates that the resolution index along boundaries of the specimen is worse $(\sim 0.8)$, thus delimiting the confidence area.

\subsection{Spatio-temporal images}

Following the above mentioned inversion procedure, we produce a series of images of velocity and structural changes for each loading time (please refer to Movie S1). Figure 5(b) presents images of 3 different physical changes at 6 successive loading times (from $75 \mathrm{~s}$ to $825 \mathrm{~s}$ ) associated to the maximum and minimum applied stress as well as initial (0 s) and final state of the sample (900 s). We also provide the loading history from stress and displacement curves (Fig. 5(a)). 


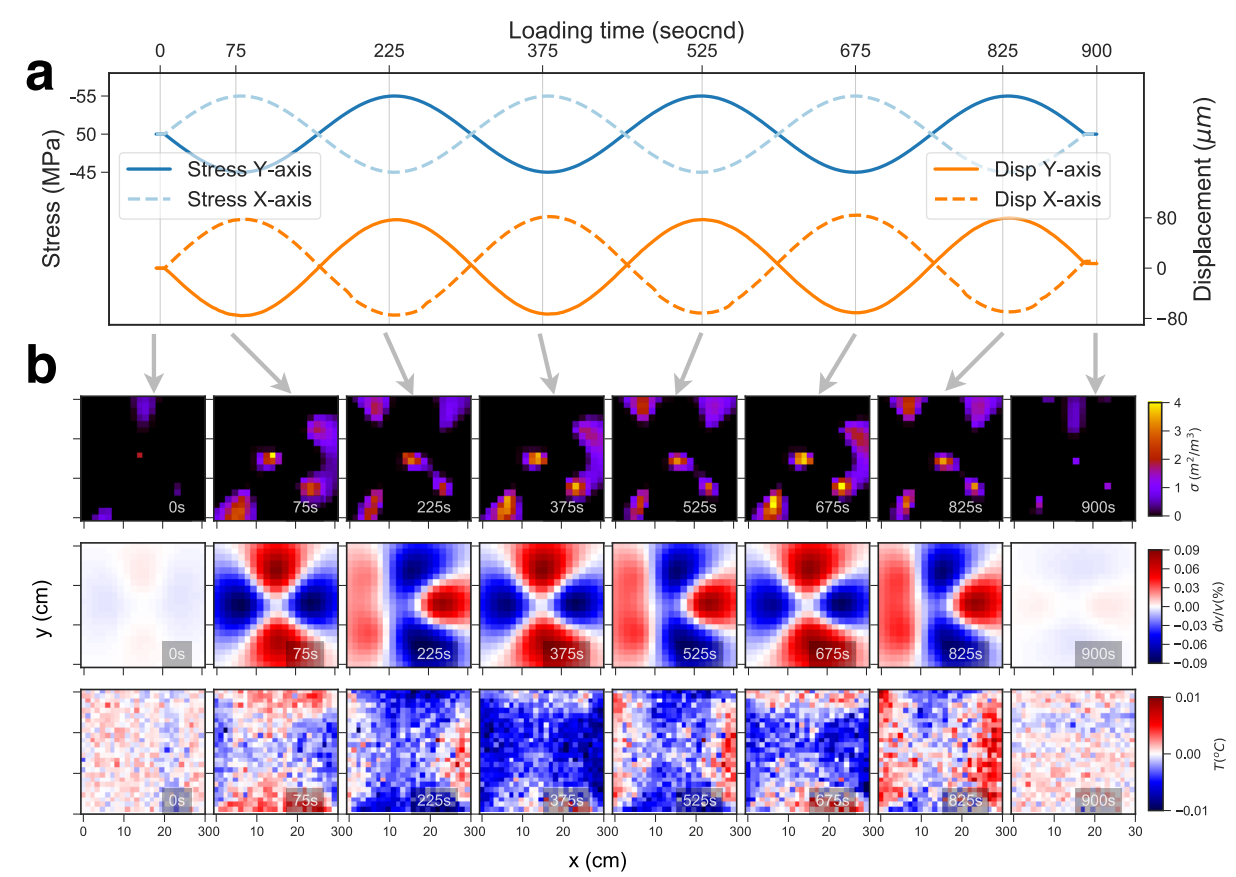

Figure 5. Snapshots of spatio-temporal images of changes in the X-Y plane at eight successive loading times from $0 \mathrm{~s}$ to $900 \mathrm{~s}$. (a) Loading history: stress and displacement curves; (b) the first row corresponds to images of micro-structural changes $(\sigma)$; the second row corresponds to images of velocity changes $(d v / v)$; the third row corresponds to infrared thermography snapshots $\left({ }^{\circ} \mathrm{C}\right)$.

The first row in Fig. 5(b) presents the distribution of structural changes $\sigma$. The images reveal that the micro-structural variation are occurring at the center and along the diagonals of the specimen. The distribution is relatively uneven, though reproducible for various loadings along the $X$ (resp. $Y$ ) direction at time $75 \mathrm{~s}, 375 \mathrm{~s}$ and $675 \mathrm{~s}$ (resp. $225 \mathrm{~s}, 525 \mathrm{~s}$ and $825 \mathrm{~s})$. Please note that the physical unit $\left(\mathrm{m}^{2} / \mathrm{m}^{3}\right)$ represents the density of scattering cross-sections of structural changes, that can be interpreted as a density of $2 \mathrm{D}$ cracks in a $3 \mathrm{D}$ cell in the case of developing damage, or to local geometrical deformation. We observe that $\sigma$ mainly concentrates at the center and along the diagonals. From a simple mechanical model considering the opposite-phase loading procedure and the geometry of the sample, we can determine that most shear deformation concentrates at the center and along the diagonals of the specimen (see later the numerical model), which is consistent with the observed $\sigma$. It is inferred that the shear deformation is developing at it's early stage by means of coalescence of micro-cracks under such loading pattern. It is, therefore, reasonable to suggest that the damage caused by the loading could be in- 
creased along these diagonals, and more specifically that the diagonals gets weaker with the experience such that the damage increases with a similar spatial pattern during the experiment. We find that the maximum value of scattering cross-section $\sigma$ reaches $4 \mathrm{~m}^{2} / \mathrm{m}^{3}$. This number has to be compared to previous works during mechanical experiments using intact concrete [Larose et al., 2015b; Zhang et al., 2016] that found structural changes of the order of $\left(0.1 \sim 1 \mathrm{~m}^{2} / \mathrm{m}^{3}\right)$ for cracks developing in concrete. In the case of granite, there exist no specific experiment to compare to, so it is hard to discriminate which part of $\sigma$ is due to reversible micro-deformation, and which part of $\sigma$ is due to irreversible damage/micro-cracking developments. But we can definitely conclude that the distribution of $\sigma$ is a quantity that perfectly images a mix of micro-deformation and micro-damage. Although it is an on-going research topic, we can anticipate that there seems to be a direct relation between the scattering cross-section $\sigma$ and the size of developing cracks [Planès et al., 2014; Xie et al., 2018a], we do notice that in general a larger scattering cross-section corresponds to greater crack dimensions (or greater cracks concentrations).

The second row in Fig. 5(b) presents the distributed evolution of velocity changes $(d v / v)$. The images reveal that the velocity perturbations occur mainly in four regions which are divided by the diagonals. The velocity change increases in upper and lower conical regions while decreases in left and right conical regions at $75 \mathrm{~s}, 375 \mathrm{~s}$ and $675 \mathrm{~s}$, accordingly with increased/decreased stress areas obtained from simple mechanical modelisation (see later the numerical model). A negative velocity change occurs in the upper and lower conical regions at $225 \mathrm{~s}, 525 \mathrm{~s}$ and $825 \mathrm{~s}$, but the spatial distribution of increased velocity (stress) is not exactly what theory would predict. This feature is understood as experimental imperfection in the loading apparatus/design at active pistons, consistently with observed strongest perturbation ("noises") of velocity changes originating from R6 and R8 which are located at the left top and bottom corner of sample (Fig. 3(b)) and the IR observations (see below). Only the regions along diagonals show zeros (white) velocity change.

To better understand and confirm the results of time-lapse stress distribution from CWI, we simultaneously monitor the surface temperature with infrared thermography (third row in Fig. 5). The presented temperature images are found to increase in upper and lower conical regions while decreasing in left and right conical regions at $75 \mathrm{~s}, 375 \mathrm{~s}$ and $675 \mathrm{~s}$. A negative temperature change occurs in such four regions at $225 \mathrm{~s}, 525 \mathrm{~s}$ and $825 \mathrm{~s}$, consistently with loading distribution (stress decrease). These results confirm that 
both velocity and temperature changes are jointly related to the bulk stress (confining pressure, see later) while the specimen remains in its elastic regime. Thus, as confirmed by IR camera, we conclude that CWI together with the Locadiff inversion technique provide cartographies of localized stress evolution of the material at test.

\section{Discussion}

The images of spatio-temporal changes raise at least two questions. First: why velocity and temperature changes are related positively to each other? Second: why does the spatial distribution of scattering cross-section $\sigma$ significantly differ from velocity or temperature changes?

To answer the first question, it is widely known from laboratory and field experiments that elastic wave velocities vary with the level of applied stress, a phenomenon known as acousto-elasticity. In our experiment, diffuse wave frequencies range from 300 $\mathrm{kHz}$ to $900 \mathrm{kHz}$, the associated wavelengths are equivalent to the mesoscopic scale of brittle rocks (grain size), leading to strong multiple scattering at grain boundaries. With the applied forces, the opening and closing of grain boundaries and grain contacts emit thermal infrared radiations [Wu et al., 2006; Chen et al., 2015]. In other word, velocity and infrared radiation depend on micro-cracks opening/closing induced by stress. This explains that images of velocity changes are similar to those from infrared thermographic ones. However the temperature images have lower sensitivity ( $\sim 5 \mathrm{mK})$ yielding to a level of detection of stress of $5 \mathrm{MPa}(1.03 \mathrm{mK} / \mathrm{MPa})$. In addition, due to the thickness of the specimen, heterogeneities and intrinsic dissipation also lead to a lower emitting efficiency of infrared radiation. This favors using ultrasonic CWI and Locadiff for future experiments instead of IR camera. What is clear, nevertheless, is that the velocity changes measured from diffuse ultrasound propagation and surface temperature changes measured from infrared thermography are both correlated to the changes of elastic properties of the material.

Concerning the spatial distribution of stress, temperature and velocity changes, a simple mechanical model which is well documented in literatures (e.g. [Karato, 2012]) is illustrated in Fig. 6 to better understand the stress distribution. By maintaining the constant pressure at $50 \mathrm{MPa}$, there should be no volumetric deformation along the X-Y plane while the loading forces are canceled out in two directions. It suggests that the specimen 

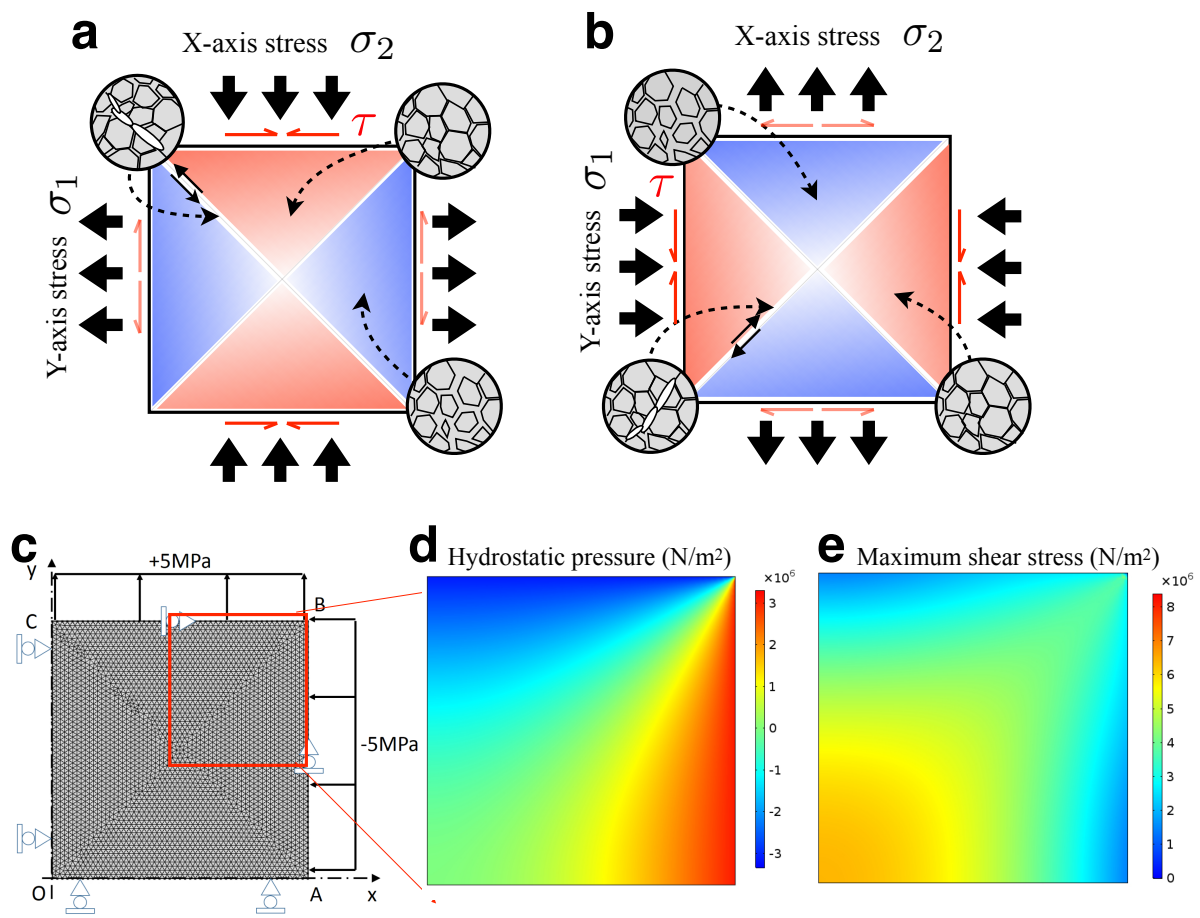

Figure 6. A simple mechanical model for understanding the stress distribution under biaxial oppositephase load. The red arrows indicate the shear stresses $(\tau)$ due to the generation of side frictions. Conical compression/tension regions associated with applied loading/unloading in (a) X-axis direction in associate with the closing (upper-right inset) and the opening (lower-right inset) contacts of grain boundaries and micro-cracks coalesce (upper-left inset) or (b) Y-axis direction in associate with the closing (lower right inset) and the opening (upper-left inset) contacts of grain boundaries and micro-cracks coalesce (lower-left inset) are formed in the bulk of the specimen during cyclic load with a 90-degree phase difference. (c) The finite element model of the sample is meshed with 6282 quadratic triangular elements. The boundary conditions under consideration are normal pressure of $-5 \mathrm{MPa}$ and $5 \mathrm{MPa}$ applied respectively to $\mathrm{AB}$ and $\mathrm{BC}$ surfaces. The nodes of the OA and CB surfaces are constrained depending to the $X$ direction, the nodes of the OC and $\mathrm{AB}$ surfaces are constrained depending to the $Y$ direction. (d) the stationary elastic behavior of upper-right quarter $(150 \mathrm{~mm} \times 150 \mathrm{~mm})$ of the rock sample under plane stress during biaxial loading. (e) the maximum shear stress of upper-right quarter $(150 \mathrm{~mm} \times 150 \mathrm{~mm})$ of the rock sample under plane stress during biaxial loading.

remains in shear state during such loading pattern and no $d v / v$ should be observed. We observe strong velocity and temperature heterogeneities that we assume due to frictions in contact surfaces between edges of the specimen and the plate of active load pistons. 
In order to verify this hypotheses on friction, we use the finite element code COMSOL to simulate the stationary elastic behavior of the upper-right quarter $(150 \mathrm{~mm} \times 150 \mathrm{~mm})$ of the rock sample under plane stress during biaxial loading. The deformable body is meshed with 6282 quadratic triangular elements. The boundary conditions under consideration are normal pressure of $-5 \mathrm{MPa}$ and $5 \mathrm{MPa}$ applied respectively to $\mathrm{AB}$ and $\mathrm{BC}$ surfaces. To mimic friction at the boundaries, the nodes of the OA and CB surfaces are constrained depending to the $X$ direction, the nodes of the OC and AB surfaces are constrained depending to the $Y$ direction. Young's modulus $E$ and Poisson's coefficient are respectively imposed to $50 \mathrm{GPa}$ and 0.3 (approximative values). In Fig. 6(c), we plot the mesh used in the numerical model. Figure 6(d) illustrates the confining pressure that results from the loading with friction at the boundaries. This numerical simulation perfectly confirms the results obtained from $d v / v$ images.

Conical compression/tension regions associated to applied loading/unloading in the Y-axis direction (Fig. 6(a)) or in the X-axis direction (Fig. 6(b)) are formed in the bulk of the specimen during cyclic loading with a 90-degree phase difference. Shear state only remains at stress junction regions e.g. along the diagonals of the specimens in our case. It is noted that the regions of velocity changes may become more irregular in shapes later in the experiment (e.g. at 825 second of loading time) because of experimental imperfection in the loading apparatus/design (e.g.frictions may increase over several periods of cyclic loadings at contact areas against active pistons while remaining constant at two other sides).

For the second question on spatial distribution, the opening and closing of microcracks not only modify the arrival times of the diffuse waves (e.g. the apparent rigidity of the material) but also the wave coherence. Considering the loading condition, the activations of micro-cracks and/or grain boundaries are related to the distribution of stress. This effect is localized mostly at places where the deformation is the greatest. In our experiment, the expected greatest local shear deformations are distributed along the diagonals (see the results of the numerical simulation of maximum shear stress Fig. 6(e)). This suggests that the development of micro-cracks and/or micro-deformation is favored by Xshape distribution of shear stress and cracks are more likely to coalesce and localize along these diagonals. Since only $5 \mathrm{MPa}$ stress perturbation is applied, and structural changes $\sigma$ mostly reversible, we believe that the damage level of the specimen is at it's early stage. The localized micro-cracks reversibility should be understood as following slow dynamics 
phenomena [Tencate et al., 1999; Guyer and Johnson, 1999]. Continuing several loading cycles may lead to micro-crack coalescence and the development of macroscopic cracks, and irreversible damage, especially along those diagonals.

Recently, laboratory observations were conducted by monitoring temporal changes in ultrasonic wave speed (coda of $\mathrm{Vp}$ ) along experimental faults throughout the seismic cycle for the complete spectrum of slip behaviors [Scuderi et al., 2016; Tinti et al., 2016]. The results show a systematic Vp reduction of $1 \%$ prior to failure (Fig. 7(a)) during the earthquake preparatory phase (weakening and rupture nucleation), spanning a wide spectrum of slip rates. Such systematic precursory variations of elastic properties for both slow and fast earthquakes are indicating similar physical mechanisms which relate to changes in asperities' contact stiffness, crack density, and disruption of asperities' force chains of the fault during rupture nucleation [Scuderi et al., 2017]. Xie et al. [2017b] also conducted a laboratory observation of stick-slip failure on 1.5-meter granite fault by measuring temporal velocity changes with a $10^{-6}$ relative resolution of diffuse ultrasound. The results of velocity reductions prior to failure (Fig. 7(b)) are consistent with previous laboratory studies using changes of $\mathrm{Vp}$. Meanwhile, a reduction in velocity of diffuse ultrasound of about $0.02 \%$ is consistent with field examples of precursory changes in seismic wave speed, such as those observed along the San Andreas Fault using seismic ambient noise (Fig. 7(c))

[Brenguier et al., 2008].

It has been demonstrated that multiple scattering (reflected) waves could be reconstructed in both active pulse-echo configuration(e.g. laboratory ultrasound experiment and/or active source field monitoring) and passive cross correlation configuration (e.g. seismic ambient noise) [Larose et al., 2006]. Due to the increasingly importance of understanding evolution in fault zone and finding clear precursors to the earthquake, the timelapse tomography method based on diffuse waves could offer a promising high-sensitive means to study the spatiotemporal evolution of elastic properties as well as the microstructures in the fault-loading medium and to detect earthquake precursors in both smallscale laboratory experiments and field experiments. 

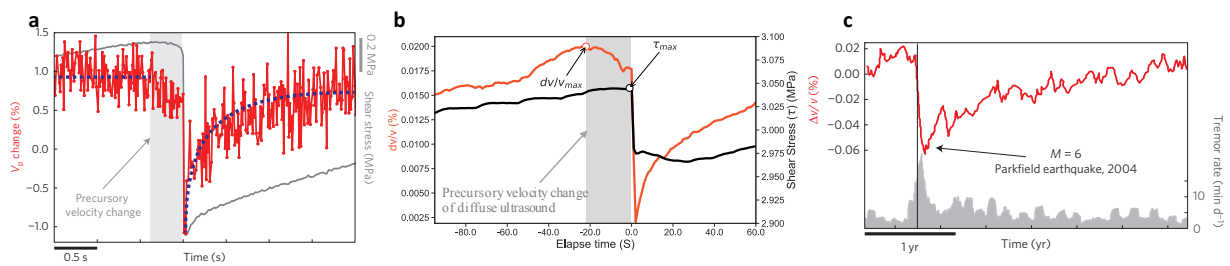

Figure 7. Comparison between laboratory and natural variation in temporal velocity change. (a) $\mathrm{Vp}$ changes during the earthquake cycle for fast audible laboratory earthquakes [modified from Scuderi et al. [2016]]; (b) velocity changes of diffuse waves during the earthquake cycle for audible laboratory earthquakes on 1.5-meter granite fault [modified from Xie et al. [2017b]]; (c) San Andreas Fault using seismic ambient noise [modified from Brenguier et al. [2008]]

\section{Conclusion and perspective}

In this study, with the application of high sensitivity diffuse ultrasound and its timelapse inversion method Locadiff, we investigated spatio-temporal mechanical changes in a heterogeneous specimen of a natural granite sample under biaxial loading.

The present work:

(1) provides a validation of the ability of the diffuse ultrasonic method to produce time-lapse images as a way to monitor stress-induced velocity changes during complex mechanical loading. By means of infrared thermography, which allowed to cartography stress induced temperature changes, we validated the results obtained from Locadiff, and demonstrated the good resolution and sensitivity of the diffuse ultrasonic technique for laboratory applications;

(2) demonstrates the detection capability to image the opening/closing of microcracks (or grain boundaries activations) and located the deformation process at early stage of damage (in a mostly reversible regime);

(3) demonstrates that a good resolution can be achieved with only a few properly distributed receivers together with one single source on one side (free surface) of the specimen.

The most important advantages of Locadiff compared to other experimental approaches is its highly sensitivity to weak changes. Also it demands a limited number of transducers and can be performed either at laboratory scale or in field experiments at seis- 
mological scales [Poupinet et al., 1984; Wang et al., 2012]. Combining such experimental method with other complementary approaches will allow to enhance the ability to investigate the mechanisms of natural rocks at mesoscopic scales under complex mechanical loading, such as laboratory earthquakes. In addition, compared to the multiple sources setup, the temporal resolution has been increased here thanks to the single source used, while spatial resolution could be maintained by using more time-windows of diffuse coda waves. Considering such advantages, further works applying Locadiff to temporal critical observations, such as nucleation process of laboratory earthquake, are conceivable from a practical and instrumental point of view.

\section{Acknowledgments}

We are grateful to the Editor A. Revil, the Associated Editor A. Schubnel, the Reviewer M.M. Scuderi and an anonymous Reviewer for comments and suggestions that significantly improved the manuscript.

We thank Peixun Liu(IG-CEA), Shunyun Chen(IG-CEA), Baoshan Wang(IGP-CEA), Zhigang Peng(Gatech) for the fruitful discussions. The data for this paper are available by contacting the corresponding author at xiefan@cea-igp.ac.cn. This research is supported by State Key Laboratory of Earthquake Dynamics[grant number LED2015B02] and National Natural Science Foundation of China [grant number NSFC41504044].

\section{References}

Aki, K. (1969), Analysis of the seismic coda of local earthquakes as scattered waves, $J$ Geophys Res, 74(2), 615-631.

Brenguier, F., M. Campillo, C. Hadziioannou, N. Shapiro, R. M. Nadeau, and E. Larose (2008), Postseismic relaxation along the san andreas fault at parkfield from continuous seismological observations, Science, 321(5895), 1478-1481.

Charalampidou, E.-M., S. A. Hall, S. Stanchits, G. Viggiani, and H. Lewis (2014), Shearenhanced compaction band identification at the laboratory scale using acoustic and fullfield methods, International Journal of Rock Mechanics and Mining Sciences, 67, 240252.

Chen, S., P. Liu, Y. Guo, L. Liu, and J. Ma (2015), An experiment on temperature variations in sandstone during biaxial loading, Physics and Chemistry of The Earth, pp. 3-8. 
Collettini, C., G. Di Stefano, B. Carpenter, P. Scarlato, T. Tesei, S. Mollo, F. Trippetta, C. Marone, G. Romeo, and L. Chiaraluce (2014), A novel and versatile apparatus for brittle rock deformation, International journal of rock mechanics and mining sciences, $66,114-123$.

Egle, D. M. (1981), Diffuse wave fields in solid media, The Journal of the Acoustical Society of America, 70(2), 476-480, doi:10.1121/1.386791.

Grêt, A., R. Snieder, R. C. Aster, and P. R. Kyle (2005), Monitoring rapid temporal change in a volcano with coda wave interferometry, Geophys Res Lett, 32(6).

Guyer, R. A., and P. A. Johnson (1999), Nonlinear mesoscopic elasticity: Evidence for a new class of materials, Physics today, 52(4), 30-36.

Hadziioannou, C., E. Larose, O. Coutant, P. Roux, and M. Campillo (2009), Stability of monitoring weak changes in multiply scattering media with ambient noise correlation: Laboratory experiments, The Journal of the Acoustical Society of America, 125(6), 36883695.

Hall, S. A. (2009), When geophysics met geomechanics: Imaging of geomechanical properties and processes using elastic waves, in Mechanics of Natural Solids, pp. 147-175, Springer.

Hansen, P. C. (1992), Analysis of discrete ill-posed problems by means of the 1-curve, SIAM review, 34(4), 561-580.

Hirsekorn, S. (1982), The scattering of ultrasonic waves by polycrystals, The Journal of the Acoustical Society of America, 72(3), 1021-1031, doi:10.1121/1.388233.

Karato, S.-i. (2012), Deformation of earth materials: an introduction to the rheology of solid earth, Cambridge University Press.

Larose, E., and S. Hall (2009), Monitoring stress related velocity variation in concrete with a $2 \times 10-5$ relative resolution using diffuse ultrasound, The Journal of the Acoustical Society of America, 125(4), 1853-1856.

Larose, E., G. Montaldo, A. Derode, and M. Campillo (2006), Passive imaging of localized reflectors and interfaces in open media, Applied physics letters, 88(10), 104103.

Larose, E., T. Planes, V. Rossetto, and L. Margerin (2010), Locating a small change in a multiple scattering environment, Appl Phys Lett, 96(20), 204101.

Larose, E., S. Carriere, C. Voisin, P. Bottelin, L. Baillet, P. Gueguen, F. Walter, D. Jongmans, B. Guillier, S. Garambois, et al. (2015a), Environmental seismology: What can we learn on earth surface processes with ambient noise?, Journal of Applied Geophysics, 
116, 62-74.

Larose, E., A. Obermann, A. Digulescu, T. Planès, J. Chaix, F. Mazerolle, and G. Moreau (2015b), Locating and characterizing a crack in concrete with diffuse ultrasound: A four-point bending test, Journal of the Acoustical Society of America, 138(1), 232-241.

Mainsant, G., E. Larose, C. Bronnimann, D. Jongmans, C. Michoud, and M. Jaboyedoff (2012), Ambient seismic noise monitoring of a clay landslide: Toward failure prediction, Journal of Geophysical Research, 117.

Miao, A.-L., S.-L. Ma, and Y.-S. Zhou (2010), Experimental study on frictional stability transition and micro-fracturing characteristics for anhydrite fault zones, Diqiu Wuli Xиebao, 53(11), 2671-2680.

Michaels, J. E., and T. E. Michaels (2005), Detection of structural damage from the local temporal coherence of diffuse ultrasonic signals, IEEE Transactions on Ultrasonics Ferroelectrics and Frequency Control, 52(10), 1769-1782.

Murnaghan, F. D. (1951), Finite deformation of an elastic solid, American Journal of Mathematics, 59(2), 235.

Niu, F., P. G. Silver, T. M. Daley, X. Cheng, and E. L. Majer (2008), Preseismic velocity changes observed from active source monitoring at the parkfield safod drill site, Nature, 454(7201), 204-208.

Obermann, A., T. Planes, E. Larose, and M. Campillo (2013), Imaging preeruptive and coeruptive structural and mechanical changes of a volcano with ambient seismic noise, Journal of Geophysical Research, 118(12), 6285-6294.

Obermann, A., B. Froment, M. Campillo, E. Larose, T. Planès, B. Valette, J. Chen, and Q. Liu (2014), Seismic noise correlations to image structural and mechanical changes associated with the mw 7.92008 wenchuan earthquake, Journal of Geophysical Research: Solid Earth, 119(4), 3155-3168.

Pacheco, C., and R. Snieder (2005), Time-lapse travel time change of multiply scattered acoustic waves, J Acoust Soc Am, 118(3), 1300-1310.

Planès, T., and E. Larose (2013), A review of ultrasonic coda wave interferometry in concrete, Cem Concr Res, 53, 248-255.

Planès, T., E. Larose, L. Margerin, V. Rossetto, and C. Sens-schonfelder (2014), Decorrelation and phase-shift of coda waves induced by local changes: multiple scattering approach and numerical validation, Waves in Random and Complex Media, 24(2), 99125. 
Planès, T., E. Larose, V. Rossetto, and L. Margerin (2015), Imaging multiple local changes in heterogeneous media with diffuse waves, Journal of the Acoustical Society of America, 137(2), 660-667.

Poupinet, G., W. L. Ellsworth, and J. Frechet (1984), Monitoring velocity variations in the crust using earthquake doublets: An application to the calaveras fault, california, Journal of Geophysical Research, 89, 5719-5731.

Ren, Y., J. Ma, P. Liu, and S. Chen (2017), Experimental study of thermal field evolution in the short-impending stage before earthquakes, Pure and Applied Geophysics, doi: 10.1007/s00024-017-1626-7.

Sabin, P. (1932), Acoustics and architecture, McGrawHill, New York and London.

Schubnel, A., P. M. Benson, B. D. Thompson, J. Hazzard, and R. P. Young (2006), Quantifying damage, saturation and anisotropy in cracked rocks by inverting elastic wave velocities, Pure and Applied Geophysics, 163, 947-973.

Scuderi, M., C. Marone, E. Tinti, G. Di Stefano, and C. Collettini (2016), Precursory changes in seismic velocity for the spectrum of earthquake failure modes, Nature geoscience, 9(9), 695.

Scuderi, M., C. Collettini, C. Viti, E. Tinti, and C. Marone (2017), Evolution of shear fabric in granular fault gouge from stable sliding to stick slip and implications for fault slip mode, Geology, G39033.1.

Snieder, R. (2006), The theory of coda wave interferometry, Pure Appl Geophys, 163(2-3), $455-473$.

Snieder, R., A. Grêt, H. Douma, and J. Scales (2002), Coda wave interferometry for estimating nonlinear behavior in seismic velocity, Science, 295(5563), 2253-2255.

Tarantola, A. (2006), Popper, bayes and the inverse problem, Nature Physics, 2(8), 492494.

Tarantola, A., and B. Valette (1982), Generalized nonlinear inverse problems solved using the least squares criterion, Reviews of Geophysics, 20(2), 219-232.

Tencate, J. A., E. Smith, and R. A. Guyer (1999), Nonlinear slow dynamics and memory in rocks, Journal of the Acoustical Society of America, 105(2), 1231.

Thompson, R. B. (1996), A generalized model of the effects of microstructure on ultrasonic backscattering and flaw detection, Review of Progress in Quantitative Nondestructive Evaluation, 15A, 1471-1478, doi:10.1007/978-1-4613-0383-1-192. 
Tinti, E., M. Scuderi, L. Scognamiglio, G. Di Stefano, C. Marone, and C. Collettini (2016), On the evolution of elastic properties during laboratory stick-slip experiments spanning the transition from slow slip to dynamic rupture, Journal of Geophysical Research: Solid Earth, 121(12), 8569-8594.

Tsai, V. C. (2011), A model for seasonal changes in gps positions and seismic wave speeds due to thermoelastic and hydrologic variations, Journal of Geophysical Research, 116(B4), doi:10.1029/2010JB008156.

Wang, B., H. Ge, W. Yang, W. Wang, B. Wang, G. Wu, and Y. Su (2012), Transmitting seismic station monitors fault zone at depth, Eos, Transactions American Geophysical Union, 93(5), 49-50.

Weaver, R. L., C. Hadziioannou, E. Larose, and M. Campillo (2011), On the precision of noise correlation interferometry, Geophysical Journal International, 185(3), 1384-1392.

Wu, L., S. Liu, Y. Wu, and C. Wang (2006), Precursors for rock fracturing and failurepart ii: Irr t-curve abnormalities, International Journal of Rock Mechanics and Mining Sciences, 43(3), 483-493.

Xie, F., L. Moreau, Y. Zhang, and E. Larose (2016), A bayesian approach for high resolution imaging of small changes in multiple scattering media, Ultrasonics, 64, 106-114.

Xie, F., E. Larose, L. Moreau, Y. Zhang, and T. Planes (2018a), Characterizing extended changes in multiple scattering media using coda wave decorrelation: numerical simulations, Waves in Random and Complex Media, 28(1), 1-14.

Xie, F., Y.-Q. Ren, and B.-S. Wang (2017b), Observation of stick-slips on a 1.5 m-long granite fault using ultrasonic coda waves, CHINESE JOURNAL OF GEOPHYSICSCHINESE EDITION, 60(4), 1470-1478.

Yamaoka, K., T. Kunitomo, K. Miyakawa, K. Kobayashi, and M. Kumazawa (2001), A trial for monitoring temporal variation of seismic velocity using an across system, $I s$ land Arc, 10(3), 336-347.

Zhang, Y., O. Abraham, F. Grondin, A. Loukili, V. Tournat, A. Le Duff, B. Lascoup, and O. Durand (2012), Study of stress-induced velocity variation in concrete under direct tensile force and monitoring of the damage level by using thermally-compensated coda wave interferometry, Ultrasonics, 52(8), 1038-1045.

Zhang, Y., T. Planes, E. Larose, A. Obermann, C. Rospars, and G. Moreau (2016), Diffuse ultrasound monitoring of stress and damage development on a 15-ton concrete beam, Journal of the Acoustical Society of America, 139(4), 1691-1701. 